\title{
In-situ monitoring for CVD processes
}

\author{
V. Hopfe ${ }^{a}$, D.W. Sheel ${ }^{b}$, C.I.M.A. Spee ${ }^{c}$, R.Tell ${ }^{d}$, P. Martin ${ }^{\text {e }}$, A. Beil ${ }^{\mathrm{g}}$, M. Pemble ${ }^{\mathrm{f}}$, R. Weiss ${ }^{\mathrm{i}}$, \\ U.Vogt ${ }^{\mathrm{h}}$, W. Graehlert ${ }^{\mathrm{a}}$ \\ ${ }^{a}$ Fraunhofer Institut Werkstoff- und Strahltechnik, Winterbergstrasse 28, 01277 Dresden, D \\ ${ }^{\mathrm{b}}$ Pilkington Technology Centre, Lathom, UK (current address: CVD -Technologies Ltd., Cockroft \\ building Salford University, Manchester, M5 4WT, UK) \\ ${ }^{c}$ TNO Institute of Applied Physics, P.O. Box 595, 5600 AN Eindhoven, NL \\ ${ }^{\mathrm{d}}$ Siemens Laser Analytics AB, Ringögatan 12 BOX 8910, 40273 Göteborg, SE \\ ${ }^{\mathrm{e}}$ University of Huddersfield, Huddersfield HD1 3DH, UK \\ ${ }^{\mathrm{f}}$ University of Salford, Salford M5 4WT, UK \\ ${ }^{g}$ Bruker-Saxonia Analytik GmbH, P.O. Box 42, 04301 Leipzig, D \\ ${ }^{\mathrm{h}}$ EMPA, Überlandstrasse 129, 8600 Dübendorf, $\mathrm{CH}$ \\ ${ }^{\text {i }}$ Schunk -Kohlenstofftechnik, P.O. Box 100951. 35339 Giessen, D
}

\begin{abstract}
Aiming toward process control of industrial high yield / high volume CVD reactors, the potential of optical sensors as a monitoring tool has been explored. The sensors selected are based on both Fourier transform infrared spectroscopy (FTIR) and tunable diode laser spectroscopy (NIR-DLS). The former has the advantage of wide spectral capability, and well established data bases. NIR-DLS spectroscopy has potentially high sensitivity, laser spatial resolution, and the benefits of comparatively easier integration capabilities - including optical fibre compatibility.

The proposed technical approach for process control is characterised by a "chemistry based" feedback system with in-situ optical data as input information. The selected optical sensors continuously analyze the gas phase near the surface of the growing layer. The spectroscopic data has been correlated with process performance and layer properties which, in turn establish data basis for process control.

The new process control approach is currently being verified on different industrialised CVD coaters. One of the selected applications deals with the deposition of $\mathrm{SnO}_{2}$ layers on glass based on the oxidation of $\left(\mathrm{CH}_{3}\right)_{2} \mathrm{SnCl}_{2}$, which is used in high volume production for low-E glazing.
\end{abstract}

\section{Introduction}

Efficient process control is essential to fully exploit the potential of high yield CVD processes which are key technologies in many industrial sectors and where relatively small yield changes can be reflected in significant impacts on commercial viability. Reactor operation is typically open loop, and conventional operation is driven by optimization of process parameters based on characterization of post processed material properties.

In recent years in-situ spectroscopic studies have been reported aiming at film growth monitoring, primarily in the field of basic research. However, the use of optical spectroscopy to monitor and control industrial CVD reactors is limited. This is due to a range of different technical reasons, e.g. low process compatibility of the available instrumentation, but also due to higher efforts required for special training of CVD plant operators. In addition, difficult engineering issues, such as optical adaptation without disturbing the coating process, need to be addressed. It should be noted that in many applications of advanced process control via monitoring, a major hurdle to be overcome is the 
resource required to establish a sufficiently detailed data base of results to allow effective evaluation of technical viability, and commercial benefit.

In order to address this challenge a group of European companies and Technology Institutes are collaborating. The new process control approach is currently being verified on different industrial CVD coaters. One of the selected applications comprises the deposition of $\mathrm{SnO}_{2}$ layers on architectural glass, which has been commercialised for low-E panes [1-3].

This paper will present labscale based results of recent process monitoring studies using Fourier Transform Infrared Spectroscopy (FTIR) and tunable NIR diode laser spectroscopy (NIR-DLS) and discuss the advantages and limitations for process use. The data base for process control has been established on both experimental results and chemical kinetics.

\section{Experimental}

The application of in-situ FTIR and NIR-DLS has been verified on atmospheric pressure CVD of $\mathrm{SnO}_{2}$ layers on glass based on the oxidation of dimethyl tin dichloride $\left(\left(\mathrm{CH}_{3}\right)_{2} \mathrm{SnCl}_{2} ; \mathrm{DMT}\right)$ with a mixture of oxygen and, in some experiments, with adding water. Deposition experiments simulating the real industrial process were carried out at first in a down-scaled CVD model reactor. The developed monitoring technology has been transferred to an industrial prototype continuous CVD coater being in use for manufacturing architectural K-glass. Based on both FTIR and NIR-DLS, several field trials have been performed resulting in very promising results for process control based on in-situ spectroscopic monitoring.

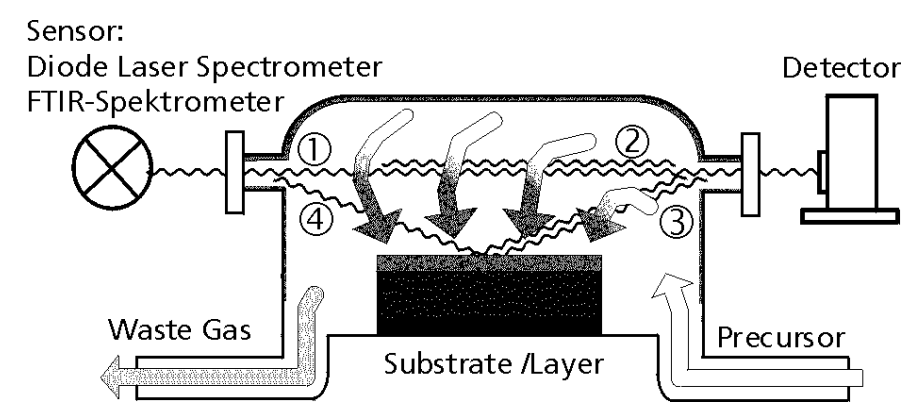

Fig. 1: Schema for optical monitoring of industrial processes using different measuring configurations: (1) transmission, (2) emission of hot gases (3) surface emission (4) reflection

For monitoring purposes the CVD reactor is equipped with two view-ports which are closed by infrared transparent windows. Four distinctly different measuring optical set-up have been applied to get information on process characteristics: transmission measurement of gas atmosphere, emissions measurement of hot gas phase, (oblique) surface emission of hot substrate combined with gas phase, and reflection measurement on coated substrate (see Fig. 1).

The FTIR investigations were performed in the spectral range from $6000 \mathrm{~cm}^{-1}$ to $350 \mathrm{~cm}^{-1}$ using both a Bruker MATRIX and a MIDAC M2500-C FTIR process spectrometer, equipped by cryogenic MCT detectors. At run-time of the CVD process (including a short interval before/after precursor flow) the FTIR runs in the „kinetic scan“ mode (time resolution: $0.5 \mathrm{~s}, 1$ scan per measurement, spectral resolution: $2 \mathrm{~cm}^{-1}$ ). To get highest sensitivity reflection and surface emission measurements are carried out using an near grazing angle of incidence of about $80^{\circ}$. A wire-grid polariser has been used at all reflection/ surface emission configurations. 
For better comparison of the measurements all single beam spectra were converted into absorbancelike units $\left(-\lg \left(\mathrm{I}_{\text {sample }} / \mathrm{I}_{\text {reference }}\right)\right.$. As a reference spectrum, those single beam spectrum has been extracted from the kinetic scan immediately before the precursor injection starts. All other reactor parameters are kept constant during deposition run, therefore only the precursor and the reaction products can be monitored in the absorbance spectrum. In case of selecting the reflection / surface emission mode, changes of the glass surface due to layer growth can be monitored additionally.

In the case where there is a high correlation of process performance with one reaction product (or a small number of several) NIR-DLS sensors can advantageously be applied. Monitoring of the gas phase by in-situ NIR-DLS have been realised by both a prototype experimental set-up [4] (detection of methane) and a modified commercial LDS3000 diode laser based $\mathrm{HCl}$ gas sensor (AltOptronic $\mathrm{AB}$ /SE). To reach a detection limit for $\mathrm{HCl}$ of about 1 ppm a multi-pass optical configuration (30 passes) has been applied.

\section{Results and Discussion}

\section{FTIR Measurements}

FTIR measurements have been performed at first to get overview on the gas phase species present within the tin oxide CVD system $[3,5,6]$. The reactive gas atmosphere during the CVD run contain non-pyrolysed DMT precursor and, in addition, a range of reaction products, as $\mathrm{CH}_{4}, \mathrm{HCHO}, \mathrm{CH}_{3} \mathrm{OH}$, $\mathrm{CO}, \mathrm{HCl}$, and $\mathrm{H}_{2} \mathrm{O}$ (see Fig. 2).

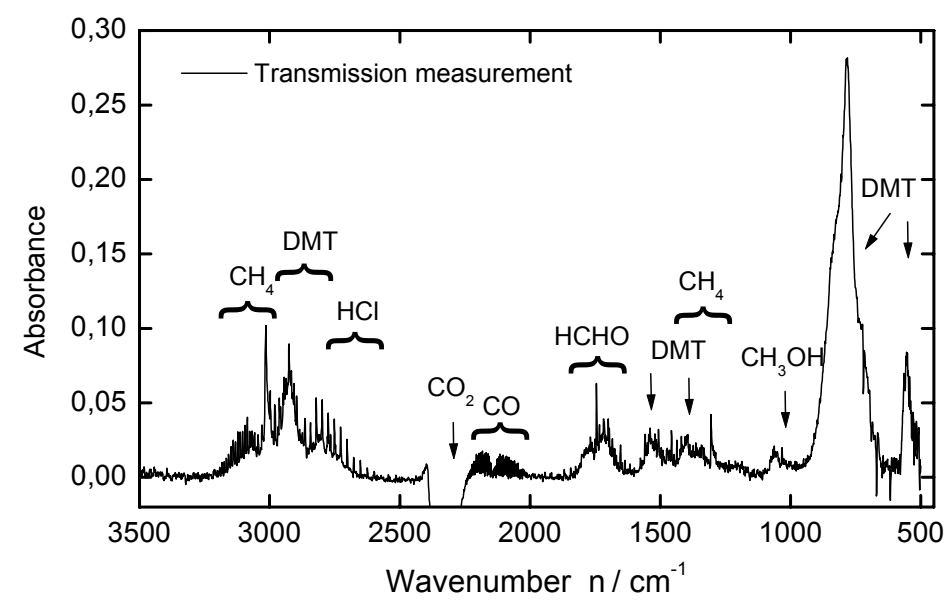

Fig. 2: Typical in-situ FTIR-absorption spectra of gas atmosphere during $\mathrm{SnO} 2$ deposition on glass using DMT- precursor

Based on FTIR-investigations at different temperatures an kinetic model of deposition process has been derived. At about $430{ }^{\circ} \mathrm{C} \mathrm{CH}_{2} \mathrm{O}$ and $\mathrm{CH}_{3} \mathrm{OH}$ are formed during deposition, but at higher temperatures their concentration decreases again and at $550{ }^{\circ} \mathrm{C}$ they are almost completely gone. The concentrations of other by-products of the deposition reaction $\left(\mathrm{HCl}, \mathrm{CO}, \mathrm{CO}_{2}, \mathrm{CH}_{4}\right.$, and $\left.\mathrm{H}_{2} \mathrm{O}\right)$ increase with reactor temperature. All these by-products show a linear increase of their concentration with the inlet concentration of DMT. Calibration of the methane signal revealed that for every mole of tin oxide exactly one mole of methane is formed.

A possible mechanism that is in accordance with the performed measurements is described below: 
Gas phase:
(1) DMT
$\rightarrow$
$\mathrm{CH}_{3} \mathrm{SnCl}_{2} \cdot+\mathrm{CH}_{3} \cdot$
(2) $\mathrm{CH}_{3} \mathrm{SnCl}_{2}$.
$\rightarrow$
$\mathrm{SnCl}_{2}$
$+\mathrm{CH}_{3}$.
(3) $\mathrm{CH}_{3}$.
$+\mathrm{O}_{2}$
$\rightarrow$
$\mathrm{CH}_{3} \mathrm{O}_{2}$.
(4) $\mathrm{CH}_{3} \mathrm{O}_{2}$.
$\rightarrow$
$\mathrm{CH}_{2} \mathrm{O}$
$+\mathrm{OH} \cdot$
(5) $\mathrm{OH} \cdot$
+ DMT $\rightarrow$
$\mathrm{CH}_{3} \mathrm{OH}$
$+\mathrm{CH}_{3} \mathrm{SnCl}_{2}$.
(6) $\mathrm{CH}_{3}$.
+ DMT $\rightarrow$
$\mathrm{C}_{2} \mathrm{H}_{6}$
$+\mathrm{CH}_{3} \mathrm{SnCl}_{2}$.

Surface:
(7) $\mathrm{O}_{2}$
$+\quad 2 *$
$\rightarrow$
2O*
(8) $\mathrm{CH}_{3} \cdot+4 \mathrm{O}^{*} \rightarrow$
$\mathrm{CO}$
$+3 \mathrm{OH}^{*}$
(9) $\mathrm{CH}_{3} \mathrm{SnCl}_{2} \cdot+3 \mathrm{OH}^{*} \rightarrow$
$\mathrm{SnO}_{2}$
$+\mathrm{CH}_{4}+2 \mathrm{HCl}+\mathrm{H}_{2} \mathrm{O}$

The mechanism is also supported by the results of the CVD monitoring experiments where a very good correlation is found between the growth of the tin oxide layer and the amount of $\mathrm{HCl}$ or $\mathrm{CH}_{4}$ in the gas phase. The concentration of gas phase species (represented by FTIR absorption intensity measurements) present during deposition has been compared with each of the experimentally determined growth rate parameters to determine any correlations. The concentration of the reaction products $\mathrm{CH}_{4}$ and $\mathrm{HCl}$ correlated nearly linearly with the total amount of tin oxide deposited over the length of the film per time unit (equivalent with deposition rate) (see Fig. 3) .
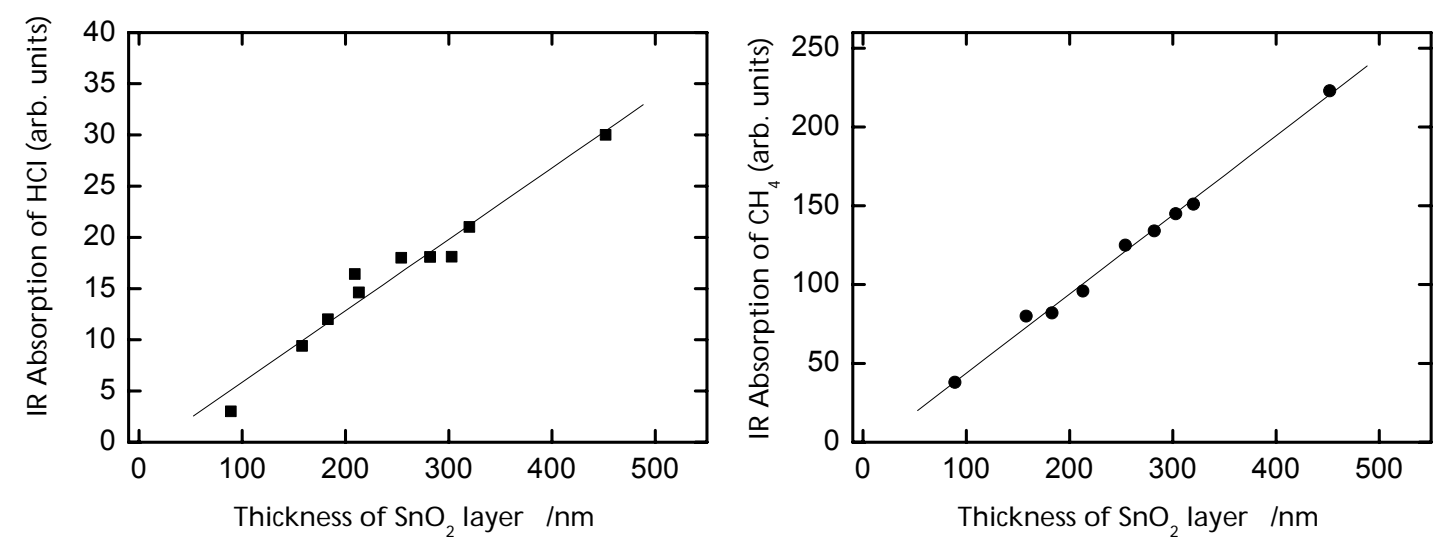

Fig. 3: Correlation of in-situ gas phase concentration (signal of FTIR absorbance) of HCl (left) and methane (right) with a measure of the thickness of deposited $\mathrm{SnO} 2$ layer

Beside the gas phase monitoring, both FTIR reflection and surface emission modes can be used for direct monitoring of surface changes, e.g. layer growth, if there is a line-of-sight toward the (aligned) substrate. Using a high angle of observation (about $80^{\circ}$ ) the plane of polarization of infrared radiation is essential for sensitivity considerations.

Unlike the transmission/emission spectra of the gas phase the spectra of surface emission and reflection are dominated by the features of the glass substrate surface (Si-O: $950 \mathrm{~cm}^{-1}, 1350 \mathrm{~cm}^{-1}$; see Fig. 4). Because of the selected background correction procedure (spectra without DMT precursor), these features do not directly arise from the growing tin oxide layer but from the decrease of the glass band. Unfortunately, the tin oxide bands $\left(620 \mathrm{~cm}^{-1}\right)$ are completely masked by the precursor (DMT) band and can not be used for monitoring of layer growth. With TM (parallel to plane of incidence) polarization, 
the simultaneous detection of reaction products is feasible but difficult to realise because of signal-tonoise constraints. In contrast to this, with TE (perpendicular) polarization the reaction product signals vanishes almost completely (first of all in surface emission mode). In either case the bands of the precursor are strong. This initially unexpected result can be explained within framework of kinetic stagnation layer approach and optical theory. Concentration of gaseous products of any surface reaction is highest near the surface. This gas layer adjacent to the surface is "scanned“ in different way by TE / TM polarised radiation. The TM wave forms a maximum of electric field strength at the surface and therefore is strongly absorbed by the gas molecules adjacent to the surface. In contrast, TE waves have zero field strength at the surface and hence are not absorbed there.

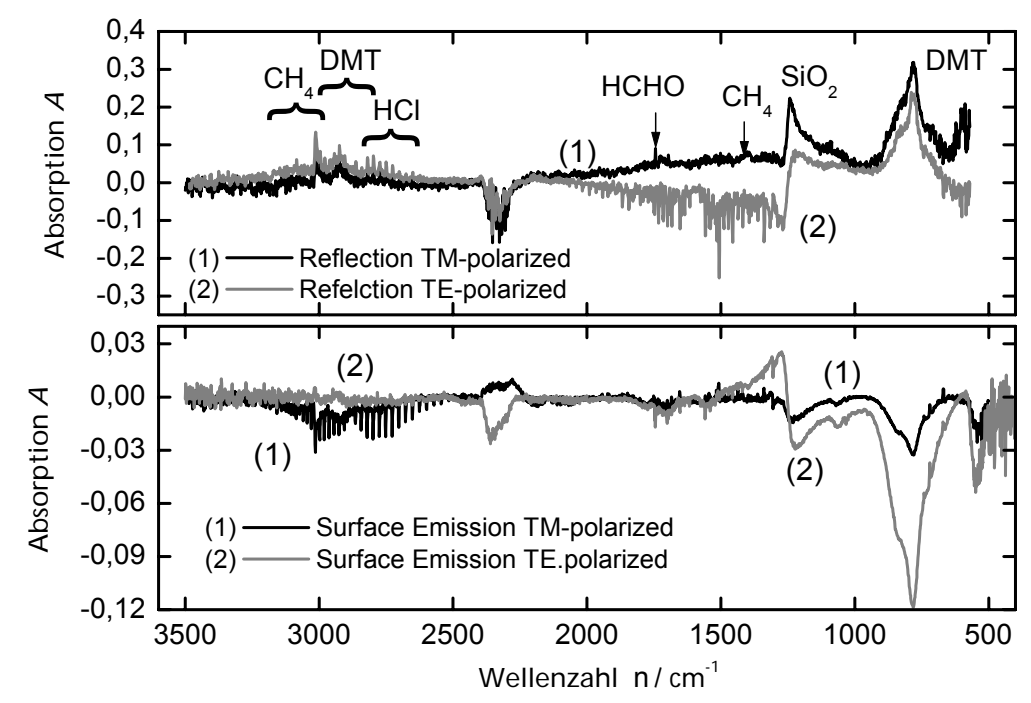

Fig 4: TE and TM polarised reflectance spectra (top) and surface emission spectra (bottom) during a typical $\mathrm{SnO} 2$ deposition run.

Glass bands correlate well with layer growth for both modes reflection and in surface emission. Obviously, the intensity change starts and stops synchronously with precursor flow. The measured thickness of all samples has been related to the peak height of the glass band and show a linear correlation (see Fig. 5). Thus, based on this simple approach of data treatment, layer thickness (and deposition rate) can be measured in real time without installing any additional sensor. The drawback of this type of combined surface-gas phase measurements compared to pure gas phase transmission/emission measurements is a considerably low signal-to-noise-ratio due to energy lost by polariser. Furthermore the optical adaptation is more complicated and needs aligned substrates.

The correlation of the glass signal intensity with the thickness of $\mathrm{SnO}_{2}$ thickness has been confirmed by optical spectra calculations using the optical model glass $/ \mathrm{SnO}_{2} /$ air. 


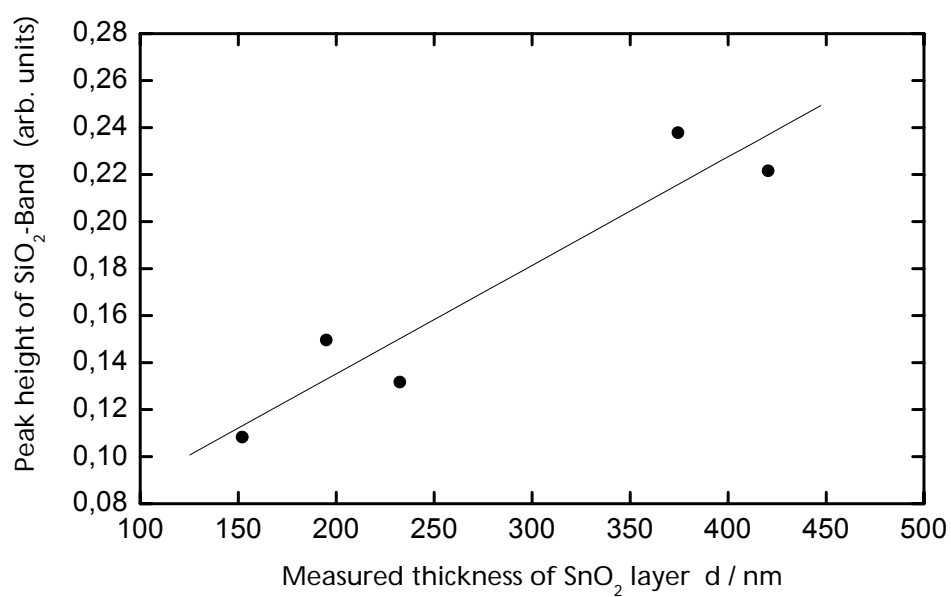

Fig. 5: Measured correlation of glass band intensity with the thickness of tin oxide layer using reflectance spectra (TM-polarised)

\section{NIR-DLS Measurement}

NIR-DLS sensors can advantageously be applied in case of having a high correlation of process performance with one reaction product (or a small number of several). As expected from FTIR results, the correlation of both the methane concentration and the $\mathrm{HCl}$ concentration inside reaction chamber adheres well to deposition rate, see Fig. 6 .
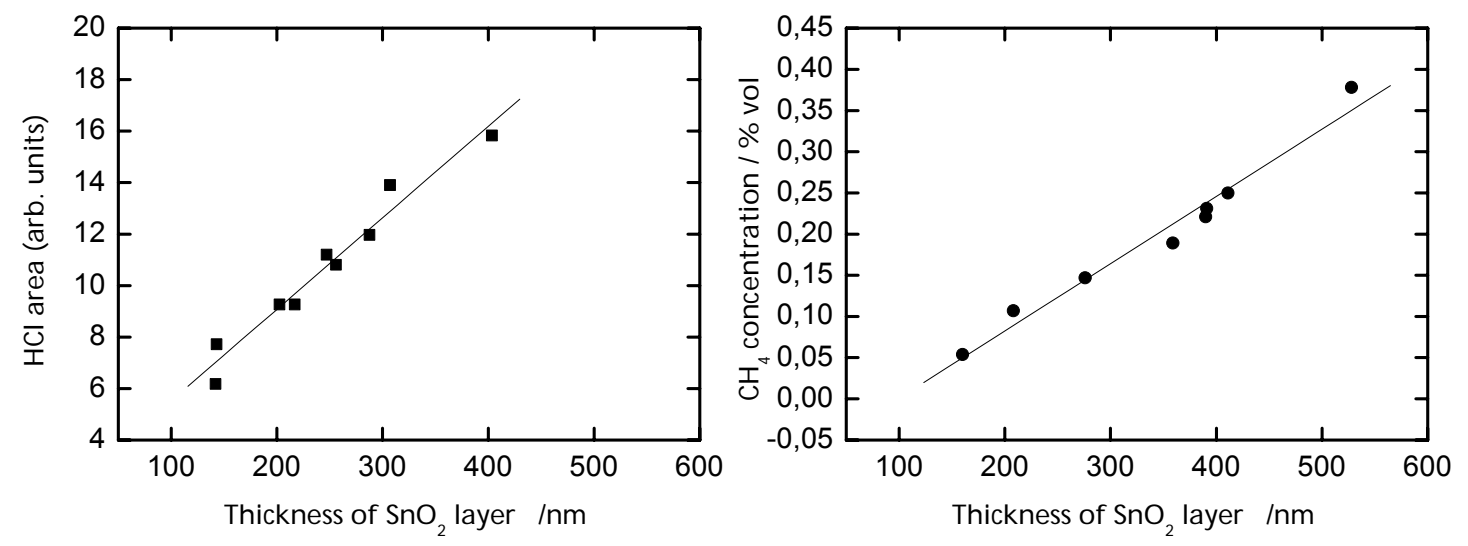

Fig. 6: Correlation of in-situ gas phase concentration (NIR-DLS signal) of $\mathrm{HCl}$ (left) and methane (right) with thickness of $\mathrm{SnO} 2$ layer (proportional to deposition rate, see text)

\section{Prediction of Process Performance}

Based on correlation data discussed above the prediction of process performance has been tested. Exploring around the parameter window for the $\mathrm{SnO}_{2}-\mathrm{CVD}$, the predicted deposition rate derived from $\mathrm{CH}_{4}$ concentrations have been compared with the experimentally determined one, see Fig. 7. The precision is much higher when compared with control of CVD reactor operation based on pre-adjusted outer parameters. 


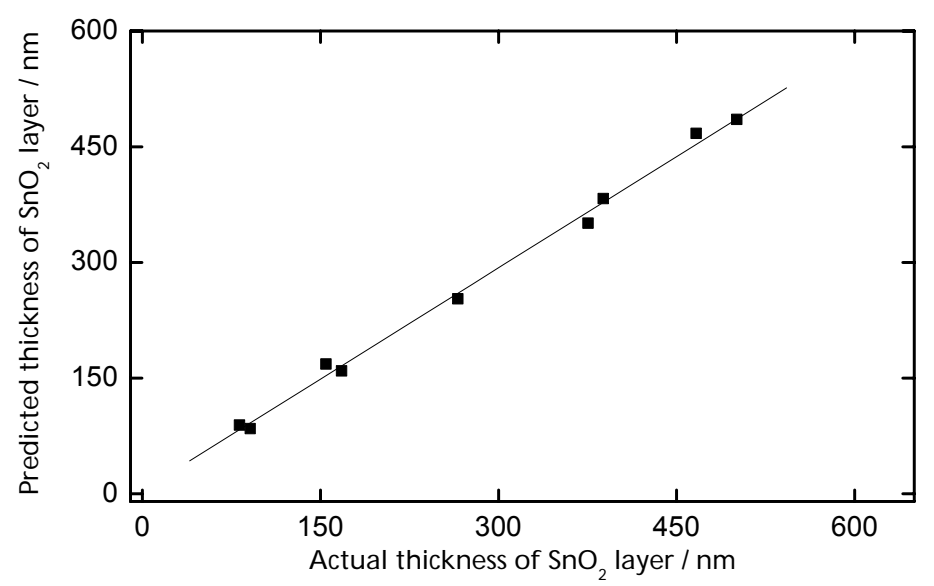

Fig. 7: Predicted thickness (calculated using the $\mathrm{CH}_{4}$ dataset measured by NIR-DLS) vs. actual thickness

Currently the in-situ spectroscopic data is being integrated into a database for automatic reactor control. The experiments have been performed at a labscale continuous CVD coater for tin oxide based on DMT $+\mathrm{O}_{2}+\mathrm{H}_{2} \mathrm{O}$ chemistry. In a first continuous trial the concentration of $\mathrm{HCl}$ has been monitored with changing precursor feed rate and, in parallel, the deposition rate have been determined (outside reactor). Again the $\mathrm{HCl}$ concentration adheres very well to the layer thickness and the deposition rate, respectively, see Fig. 8.

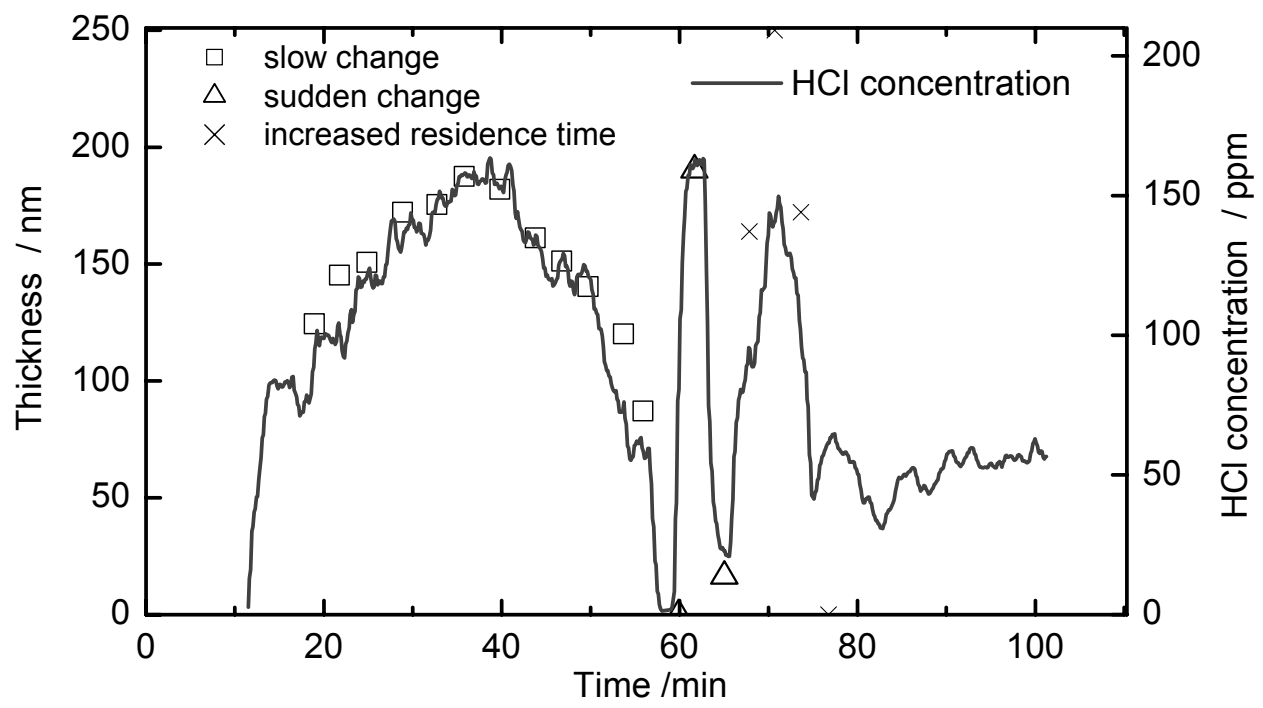

Fig. 8: $\mathrm{HCl}$ concentration and tin oxide layer thickness vs. time

The NIR- diode laser sensor has been integrated in trials running over several weeks, without any degradation of measuring performance. Currently the developed monitoring technology is being transferred to an industrial prototype continuous CVD coater being in use for manufacturing architectural glass with low emissivity properties. Based on both FTIR and NIR-DLS, several field trials have been performed resulting in very promising results for process control based on in-situ spectroscopic monitoring. The correlations are highlighting the preferential use of in-situ 
measurements which determine actual concentration of precursor and, in parallel, of reaction products. Control of mass flows feeding into reactor is not sufficient to establish an effective database for reproducible reactor control.

\section{Conclusion}

A „chemically sensitive“ CVD process control concept based on monitoring gas phase inside reaction chamber has been successfully demonstrated. Non-invasive optical sensors based on diode laser spectroscopy in the near infra red have been successfully applied for monitoring industrial CVD reactors. NIR-DLS is characterised by high sensitivity, laser spatial resolution, and the benefits of comparatively easier integration capabilities - including optical fibre compatibility. This technology has some notable potential advantages for production process applications. For example, the technology is robust and simple to operate, interference between species detection can be reduced, and simultaneous multi-point monitoring is readily achieved. Application of in-situ optical data, can lead to enhanced process performance predictability. Kinetic investigations are underway to get a more close understanding of the physico-chemical background underlying the empirically determined stiff correlation between gas phase composition and deposition rate.

Beside the glass deposition process the sensor principle have been successfully applied to $\mathrm{SiC}$ deposition on graphite by a CVD batch process [7] as well as to high temperature fibre growth process $[8]$.

\section{Acknowledgments}

This work was supported by the European Commission under a Brite Euram contract: BRPR-CT960322 .

\section{References}

[1] R. Gordon, J. Non-Cryst. Solids 218 (1997) 81-91.

[2] R. McCurdy, M. Soubeyrand, Chemical Vapor Deposition. Proc. 14th Int. Conf. and EUROCVD11, Paris 5-9 September 1997, M.D. Allendorf, and C. Bernard Eds., pp.463-70.

[3] H.E. Sanders, Chemical Vapor Deposition, Proc. 14th Int. Conf. and EUROCVD-11, Paris 5-9 September 1997, M.D. Allendorf, and C. Bernard Eds., pp.81-88.

[4] R.J. Holdsworth, P.A. Martin, D. Raisbeck, M.E. Pemble, J. Phys. IV France 9 (1999) Pr8-109

[5] V. Hopfe, W. Graehlert, O. Throl, J. Phys. IV France 9 (1999) Pr8-995

[6] D.W. Sheel, D. Raisbeck, J. Rivero, H. Sanders, J. Phys. IV France 9 (1999) Pr8-109

[7] K. Brennfleck, S. Schneweis, R. Weiss, J. Phys. IV France, 9 (1999) Pr8-1041.

[8] U. Vogt, A. Vital, W. Graehlert, V. Hopfe, M. Leparoux, H. Ewing, A. Beil, R. Daum, J. Phys. IV France, 10 (20) Pr2-43. 